\title{
AtFKBP53 is a histone chaperone required for repression of ribosomal RNA gene expression in Arabidopsis
}

\author{
Hong $\mathrm{Li}^{1}$, Sheng Luan ${ }^{1}$ \\ ${ }^{I}$ Department of Plant and Microbial Biology, University of California, Berkeley, CA 94720, USA
}

\begin{abstract}
Chromatin structure is important for controlling gene expression, but mechanisms underlying chromatin remodeling are not fully understood. Here we report that an FKBP (FK506 binding protein) type immunophilin, AtFKBP53, possesses histone chaperone activity and is required for repressing ribosomal gene expression in Arabidopsis. The AtFKBP53 protein is a multidomain FKBP with a typical peptidylprolyl isomerase (PPIase) domain and several highly charged domains. Using nucleosome assembly assays, we showed that AtFKBP53 has histone chaperone activity and the charged acidic domains are sufficient for the activity. We show that AtFKBP53 interacts with histone H3 through the acidic domains, whereas the PPIase domain is dispensable for histone chaperone activity or histone binding. Ribosomal RNA gene (18S rDNA) is overexpressed when AtFKBP53 activity is reduced or eliminated in Arabidopsis plants. Chromatin immunoprecipitation assay showed that AtFKBP53 is associated with the 18S rDNA gene chromatin, implicating that AtFKBP53 represses $r R N A$ genes at the chromatin level. This study identifies a new histone chaperone in plants that functions in chromatin remodeling and regulation of transcription.
\end{abstract}

Keywords: Arabidopsis, immunophilin, chromatin, histone chaperone, ribosomal RNA, nucleosome assembly

Cell Research (2010) 20:357-366. doi: 10.1038/cr.2010.22; published online 9 February 2010

\section{Introduction}

Chromatin in all eukaryotes is composed of basic units called nucleosomes that consist of histones and DNA. The crystal structure shows that each nucleosome contains $146 \mathrm{bp}$ of DNA wrapping around the central core histones (two copies of each of $\mathrm{H} 2 \mathrm{~A}, \mathrm{H} 2 \mathrm{~B}, \mathrm{H} 3$ and $\mathrm{H} 4$ ) [1-4]. However, the assembly and dynamics of chromatin and factors that regulate its structure are not well understood, especially in plant.

Histones are highly charged basic proteins and naturally tend to form complex aggregates that may not be suitable for chromatin assembly. Therefore a number of molecular chaperones are required to prevent them from making improper interactions and to facilitate histone deposition into chromatin structure [5-8]. Most chaperones for the core histones show preference for binding with either $\mathrm{H} 3 / \mathrm{H} 4$ or $\mathrm{H} 2 \mathrm{~A} / \mathrm{H} 2 \mathrm{~B}$. For example, nucleosome

Correspondence: Sheng Luan

Tel: +1-510-642-4995; Fax: +1-510-642-4995

E-mail: sluan@berkeley.edu

Received 16 April 2009; revised 31 August 2009; accepted 22 October 2009; published online 9 February 2010 assembly protein 1 (NAP1) or NAP1-related protein, nucleoplasmin and nucleolin preferentially bind $\mathrm{H} 2 \mathrm{~A} / \mathrm{H} 2 \mathrm{~B}$ [9-12], whereas anti-silencing function 1 (Asf1), histone cell-cycle-regulation-defective homolog A or chromatin assembly factor 1 (CAF-1) specifically interacts with $\mathrm{H} 3$ / H4 [8, 13-15]. The deposition of histone variant, such as H2A.Z, requires specific proteins as well $[16,17]$. Extensive biochemical experiments on the assembly of nucleosome in vitro were carried out to identify histone chaperones and these studies support the essential regulatory role of histone chaperones in mediating the deposition of histone onto DNA. For example, CAF-1, which is a heterotrimeric complex, is found to be generally associated with newly synthesized $\mathrm{H} 3$ and $\mathrm{H} 4$ and deposits histones on the replicating fork [18]. NAP1 is a histone chaperone that facilitates both the cytoplasm-nucleus transport of histone and its deposition into nucleosomes [19]. Recent studies also show new functions of histone chaperones in environmental adaptation in plant $[20,21]$.

It is widely believed that changes in chromatin structure alter gene expression [22-24]. Histone chaperones help maintain a delicate balance between nucleosome assembly and disassembly, thereby have profound influence on gene expression $[16,24,25]$. When chromatin 
is tightly packaged into the nucleosomes, it is not an active template for transcription. Factors that unravel nucleosomal structure often are involved in activating gene transcription. The activity of transcription generally correlates with low nucleosome density allowing for the passage of the transcriptional machine. For instance, yeast Asf1 plays a significant role in transcriptional activation of PHO family gene by the removal of nucleosomes from their promoter region [26, 27]. Deletion of $A S F 1$ or CAF-1 components led to global transcriptional misregulation, including both activation and repression, of genes functioning in a broad range such as chromatin, signal transduction and so on [28]. These studies suggest that histone chaperones function as active agents in the maintenance of chromatin dynamics and are global regulators of gene expression.

Identifying all the nuclear factors involved in nucleosome assembly will be the first step to understand the mechanism of chromatin remodeling and its effects on gene regulation. Although some histone chaperones are identified, many remain to be uncovered. As a large group of protein folding catalysts, some immunophilins have recently been shown to have a role in remodeling chromatin. The first nuclear FKBP protein, Fpr3, was isolated from $S$. cerevisiae and shown to interact with histone H2B [29]. Furthermore, SpFkbp39p and Fpr4 were identified as acidic histone chaperones in $S$. pombe and $S$. cerevisiae, respectively [7, 30]. In plants, Arabidopsis AtCYP71, a member of cyclosporine A binding proteins (cyclophilins, CYPs), was also shown to interact with histone $\mathrm{H} 3$ and regulate gene expression pattern that determines the development of Arabidopsis [31]. Analysis of these factors suggested that nuclear immunophilins might contribute to the dynamics of chromatin complex assembly and/or disassembly because they interact with basic chromatin components.

\section{Results}

\section{Domain structure of the AtFKBP53 protein}

AtFKBP53 is encoded by a single gene locus (At4g25340) in the Arabidopsis genome. This protein is a member of the FKBP-type immunophilin family in Arabidopsis [32]. It belongs to the multidomain immunophilins that contain one or more other identifiable protein motifs in addition to the catalytic peptidylprolyl isomerase (PPIase) domain. The AtFKBP53 protein contains a single FKBP domain at the $\mathrm{C}$ terminus and a highly charged $\mathrm{N}$ terminus with several regions enriched in acidic and basic amino acid residues. AtFKBP53 has three acidic regions enriched with glutamate and aspartate residues and one basic region rich in lysine residues (Figure 1). Sequence alignments show that AtFKBP53 contains similar domain structure as yeast SpFkbp39 [7]. AtFKBP53 and SpFkbp39 share the positively charged domain in the middle of the proteins, which is different from previously characterized histone chaperones containing only acidic domains. A BLAST search using AtFKBP53 protein as a query identified homologues in rice, with similar acidic, basic and FKBP domains. The similar domain structure indicates that this type of histone chaperone may be highly conserved and have similar roles in different organisms.

\section{Subcellular localization and expression pattern of At- FKBP53}

The similarity of protein structure between AtFKBP53 and yeast SpFkbp39 suggests that AtFKBP53 may have similar function with SpFkbp39. As a step toward understanding the function of AtFKBP53, we determined the subcellular localization of AtFKBP53. We fused $A t$ FKBP53 coding sequence with RFP and transformed the DNA construct into onion epidermal cells. Subcellular localization was determined using epifluorescence microscopy. As a control, we bombarded onion epidermal cells with vector containing only DNA sequence encoding the RFP protein. We found that red fluorescence was distributed in both the cytoplasm and the nucleus (data not shown). When the cells were transformed with the AtFKBP53-RFP protein fusion construct, the red fluorescence was predominantly detected in the nucleus (Figure $2 \mathrm{~A}$ and $2 \mathrm{~B}$ ), suggesting that AtFKBP53 is localized in the nucleus.

It was reported that $A t F K B P 53$ was broadly expressed in all the tissues examined, such as leaves, flowers, stems and roots [32]. To examine the detailed expression pattern of AtFKBP53 gene, we fused AtFKBP53 promoter with the GUS reporter and transformed it into Arabidopsis. Transgenic plants expressing AtFKBP53::GUS were analyzed and showed strong GUS staining in root apical meristem region and pollen (Figure 2C-2E). We compared microarray data in Genevestigator and found that AtFKBP53 expression level in the root tip is remarkably higher than other tissues examined (Figure 2F).

\section{Histone chaperone activity of AtFKBP53}

Nuclear localization of AtFKBP53 and structural similarity with other histone chaperones such as Spfkbp39 prompted us to examine whether AtFKBP53 can exert its effect as a histone chaperone and function in chromatin remodeling. We expressed and purified the recombinant proteins, SpFkbp39-His, GST, GST-AtFKBP53 and truncated forms of AtFKBP53 from E. coli. The truncated forms include GST-AtFKBP53-N1, GST-AtFKBP53-N2 


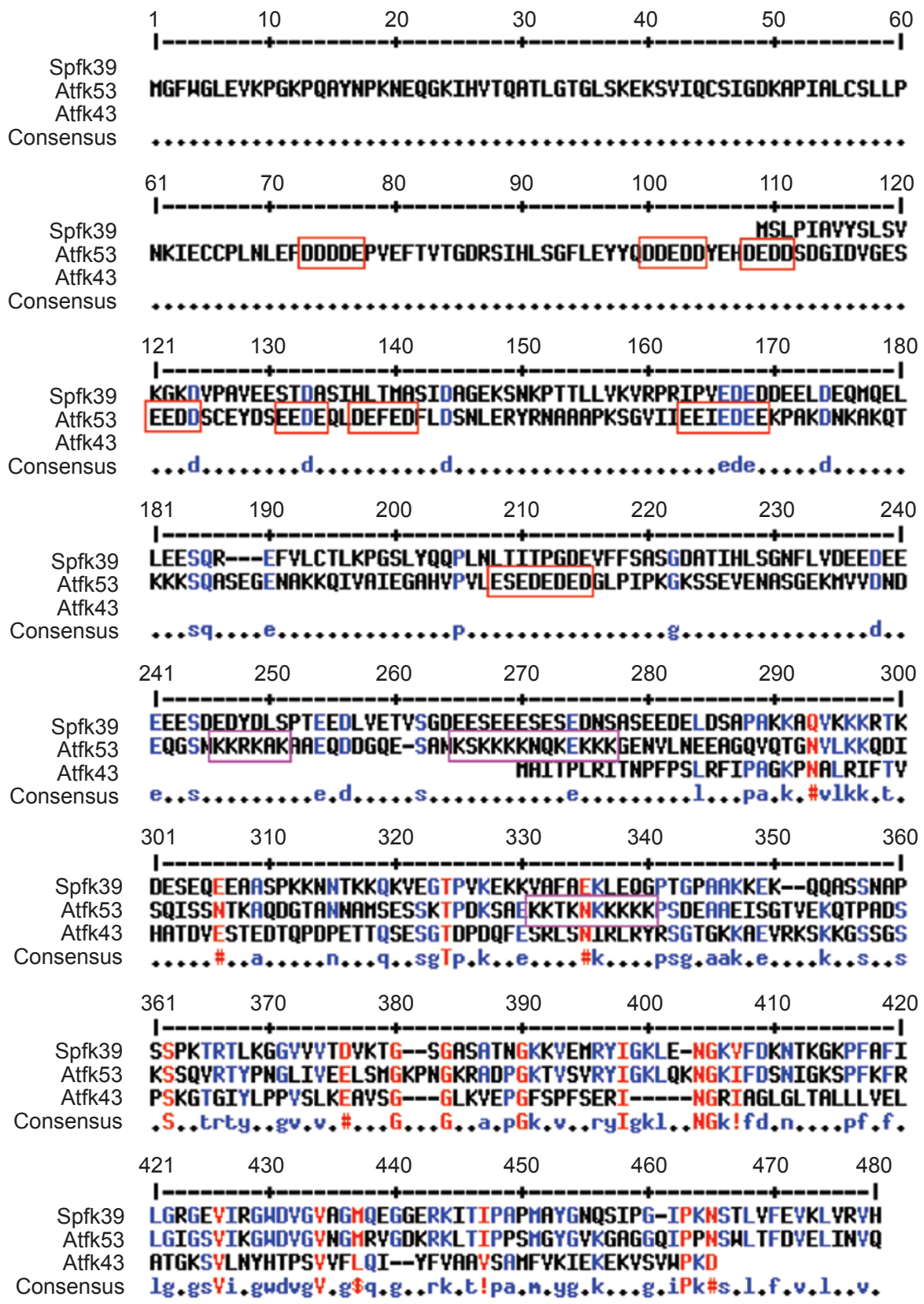

Figure 1 Sequences alignments of AtFKBP53, AtFKBP43 and SpFkbp39. Amino acids of full-length proteins of AtFKBP53, AtFKBP43 and SpFkbp39 were used for the alignment. E-, D- or K-enriched regions of AtFKBP53 are highlighted by boxes. Spfk39 is SpFkbp39, Atfk53 is AtFKBP53 and Atfk43 is AtFKBP43. Consensus amino acids among these sequences are colored.

and GST-AtFKBP53-C1, structures of which are shown in Figure 3A. Equal molar amount of protein was used for all proteins to perform plasmid supercoiling assay. SpFkbp39 was taken as a positive control, and GST as a negative control. Enhanced production of supercoiled plasmid was observed in the presence of AtFKBP53, as in the presence of SpFkbp39, suggesting that AtFKBP53, like SpFkbp39, has histone chaperone activity. When
GST or GST-AtFKBP53-C1 was used in this assay, no supercoiled plasmid was detected. However, when GSTAtFKBP53-N1 or GST-AtFKBP53-N2 was added in this assay, supercoiled plasmid was again observed (as shown in Figure 3B). Taken together, our study data indicate that AtFKBP53 possesses histone chaperone activity. The acidic domains are required and sufficient for histone chaperone activity. 

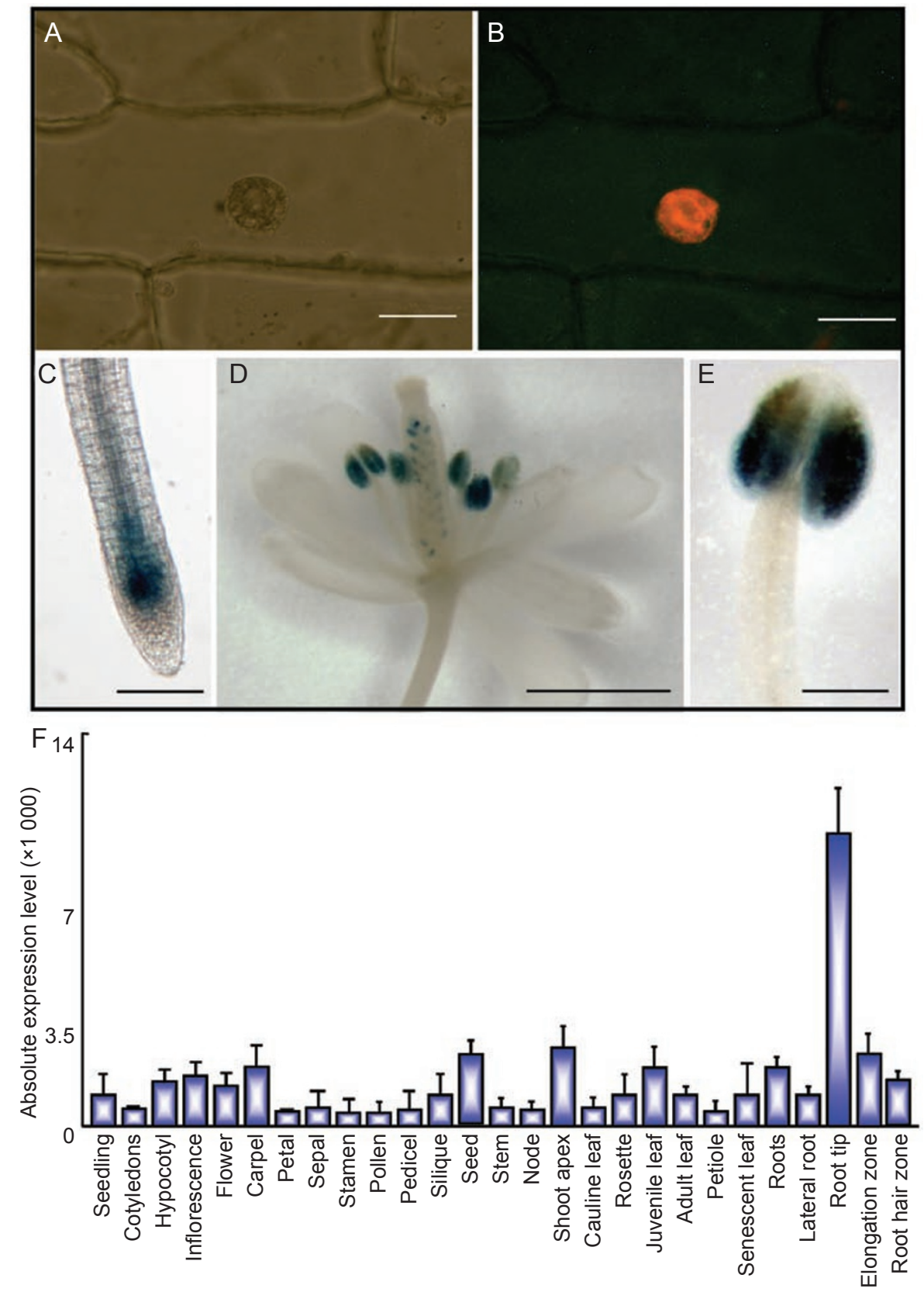

Figure 2 Subcellular localization of AtFKBP53 protein and expression pattern of the AtFKBP53 gene. (A and B) Nuclear localization of the AtFKBP53-RFP fusion protein transiently expressed in onion epidermis. (A) Bright field of an onion epidermis cell. (B) Red fluorescence of an onion epidermis cell. (C-E), Expression pattern of AtFKBP53::GUS fusion gene. (C) GUS activity in the root apical region. (D) GUS activity in the flower. (E) GUS activity in the pollen. (F) AtFKBP53 gene expression pattern. Histograms represent expression level of AtFKBP53 gene in different tissues. Bars represent standard deviation. The graph was generated based on the microarray data from Genevestigator (https://www.genevestigator.com/gv/index.jsp). Scale bars: $10 \mu \mathrm{m}$ in (A-B), $0.2 \mathrm{~mm}$ in (C) and (E), $1 \mathrm{~mm}$ in (D).

\section{AtFKBP53 interacts with histone $\mathrm{H3}$}

We showed that AtFKBP53 has histone chaperone activity and facilitates deposition of histones to DNA. To further understand how AtFKBP53 serves as a histone chaperone and the kind of histones it can directly interact with, we performed an in vitro pull-down assay using purified protein GST-AtFKBP53 with core histone proteins. GST-AtFKBP53 or GST protein was immobilized to the 
A

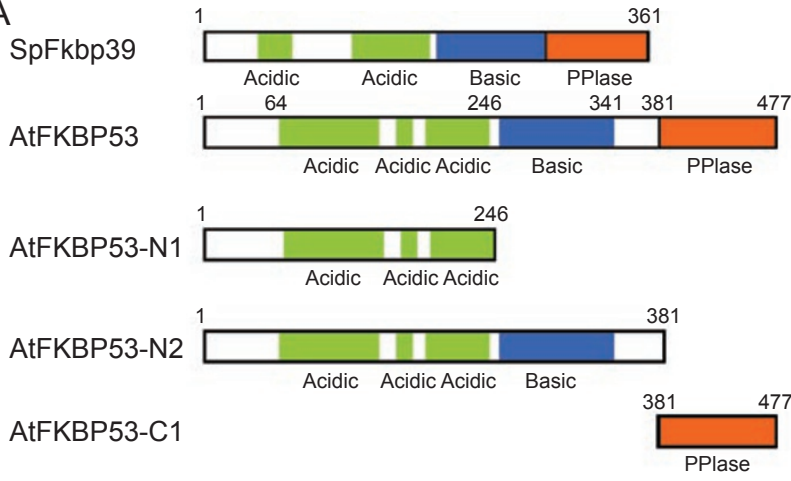

B

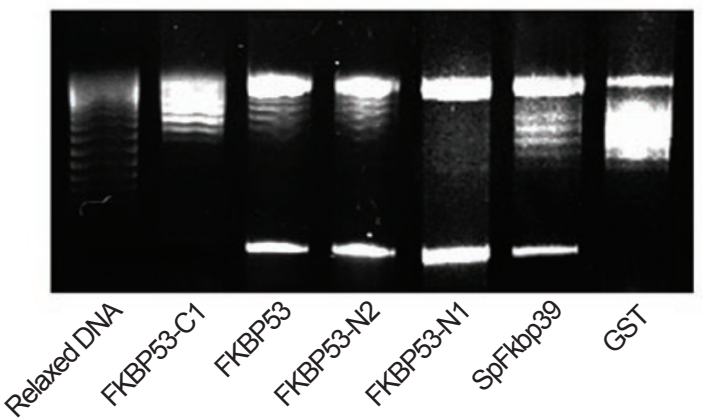

Figure 3 AtFKBP53 protein domains and histone chaperone activity assay. (A) Schematic presentation of SpFkbp39, full-length and truncated forms of AtFKBP53. (B) Plasmid supercoiling assay for different proteins. The names of protein samples are labeled under each lane.

glutathione-Sepharose beads that were further incubated with histones. The beads were washed and analyzed by SDS-PAGE. The gel was stained by Coomassie blue, which showed an obvious $17 \mathrm{kDa}$ protein precipitated specifically by AtFKBP53, not by GST. We further confirmed the precipitated protein by western blot using histone $\mathrm{H} 3$ antibody. We found that histone $\mathrm{H} 3$ was associated with AtFKBP53 in vitro, but not with GST protein. In addition, our data earlier also showed that the acidic domain is sufficient for histone chaperone activity, suggesting possible association of histone $\mathrm{H} 3$ directly to the acidic region of the AtFKBP53 protein. Further in vitro pull-down assay using truncated AtFKBP53 proteins detected histone H3 interaction with GST-AtFKBP53-N1 and GST-AtFKBP53-N2, but not with GST-AtFKBP53$\mathrm{C} 1$. This confirmed that the acidic domains are required and sufficient for binding histone $\mathrm{H} 3$ (Figure 4A and 4B).

Suppression of AtFKBP53 gene enhances $18 S$ rRNA expression

To determine the biological function of AtFKBP53, we isolated one T-DNA insertion mutant of AtFKBP53 gene from ABRC seed center. Genomic DNA analysis shows that the T-DNA was inserted between the first exon and first intron (Figure 5A). We named this T-DNA line as $f k b p 53-1$. RT-PCR analysis showed that mRNA level of AtFKBP53 was reduced in the fkbp53-1 mutant (Figure 5B). The fkbp53-1 mutant does not show obvious developmental defects under normal conditions. We further applied RNA interference technique to silence AtFKBP53 gene expression in transgenic plants. The AtFKBP53 expression was essentially eliminated in the RNA interference line. We chose one RNAi line named fkbp53-2 mutant for further studies (Figure 5C). The fkbp53-2 mutant plants, like the T-DNA insertion line, show no developmental defects.

It was previously reported that SpFkbp39 in yeast is not an essential gene but required for repressing the ribosome RNA gene expression in yeast [7]. If AtFKBP53 plays a similar function as Spfkbp39 in yeast, we would expect that $f k b p 53$ mutants may have elevated levels of $18 \mathrm{~S}$ rRNA. Indeed, in both $f k b p 53-1$ and fkbp53-RNAi
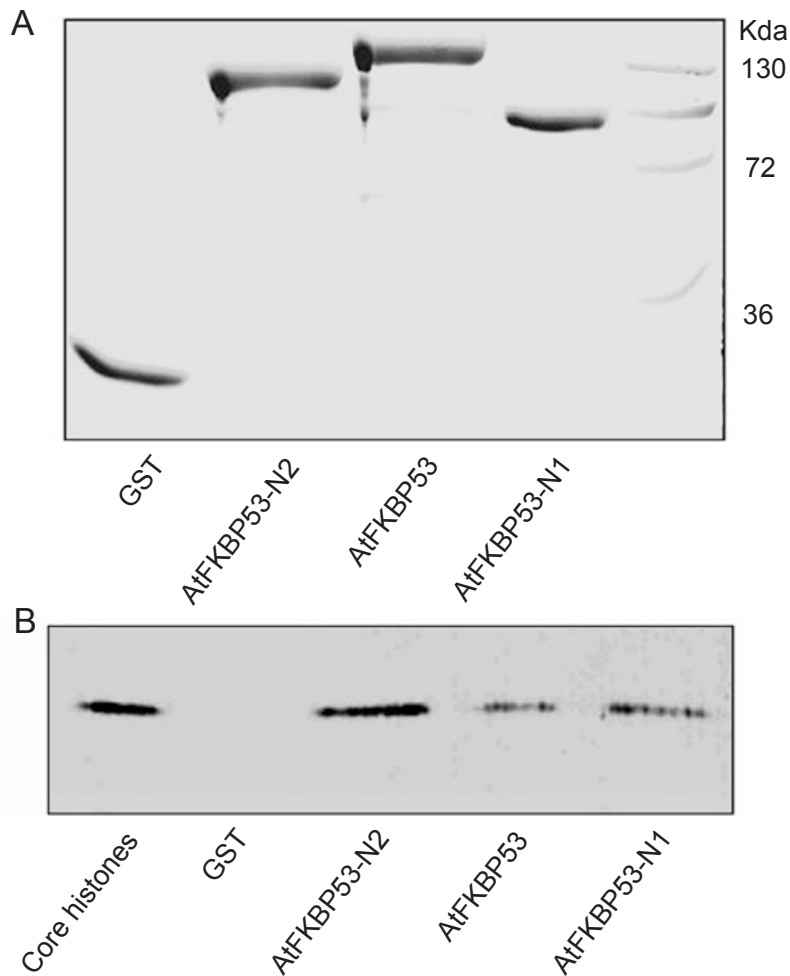

Figure 4 The AtFKBP53 protein interacts with histone H3. (A) Coomassie blue staining of GST-AtFKBP53 and GST-truncated forms ( $\mathrm{N} 1$ and $\mathrm{N} 2$ ) that were expressed and purified from $E$. coli and used to examine the interaction with the $\mathrm{H} 3$ peptide in (B). (B) The $\mathrm{H} 3$ peptide pulled down by full-length or truncated forms (N1 and N2) of AtFKBP53 was analyzed by western blot using anti-H3 antibody. 
A

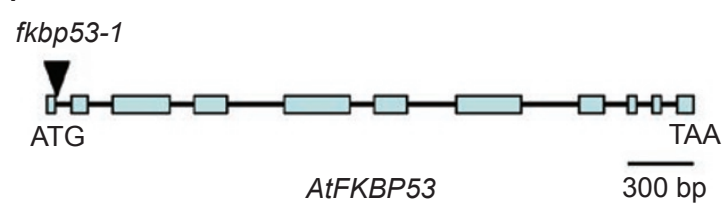

B

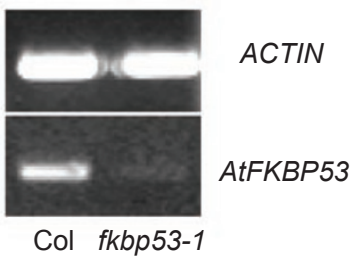

E

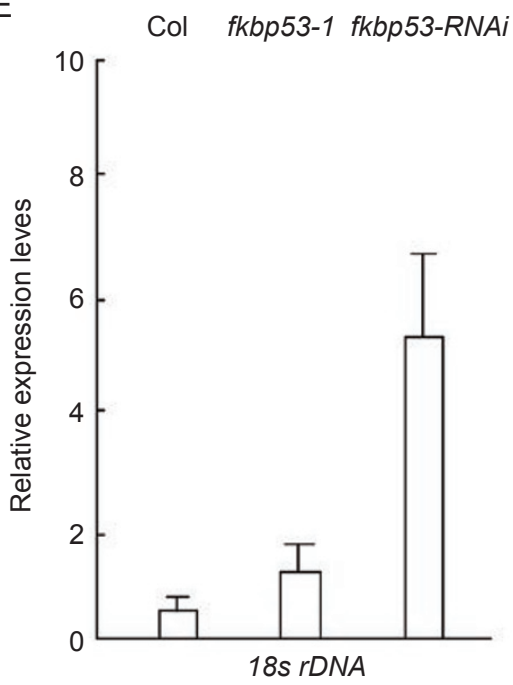

Figure 5 The fkbp53 mutants and 18S rRNA expression analysis. (A) Position of fkbp53-1 T-DNA insertion. (B) AtFKBP53 expression in wild-type and fkbp53-1 mutant. (C) AtFKBP53 expression in transgenic plants of RNAi empty vector and AtFKBP53 RNAi construct, fkbp53-2 was used to represent the transgenic plant from RNAi construct. (D) 18S rDNA expression in wild-type and fkbp53-1 mutant. (E) Quantitative PCR assay of expression level of $18 S$ rDNA in wild-type, fkbp53-1 and fkbp53-2 mutants. Bars represent standard deviation.

mutants the 18S rRNA level was higher as compared with that of wild-type plants (Figure 5D). We also quantified the expression 18S rRNA levels by real-time quantitative PCR. The results showed that $18 \mathrm{~S}$ rRNA level was increased in both $f k b p 53-1$ and $f k b p 53-R N A i$ (Figure $5 \mathrm{E})$.

\section{AtFKBP53 associates with $18 S$ rDNA chromatin}

AtFKBP53 was localized in the nucleus and in vitro pull-down assay showed that AtFKBP53 physically interacts with histone H3. To test the possibility that AtFKBP53 interacts with rRNA gene chromatin and represses rRNA gene expression, we expressed AtFKBP53-GFP fusion in transgenic Arabidopsis plants. The fusion protein was detected by western blot using GFP antibody and by examining the GFP (Figure 6A and 6B). We used the ChIP technique to examine the association of AtFKBP53 with rDNA using three independent transgenic plants expressing AtFKBP53-GFP. The ChIP assay of these lines showed that AtFKBP53 associates with $18 \mathrm{~S}$
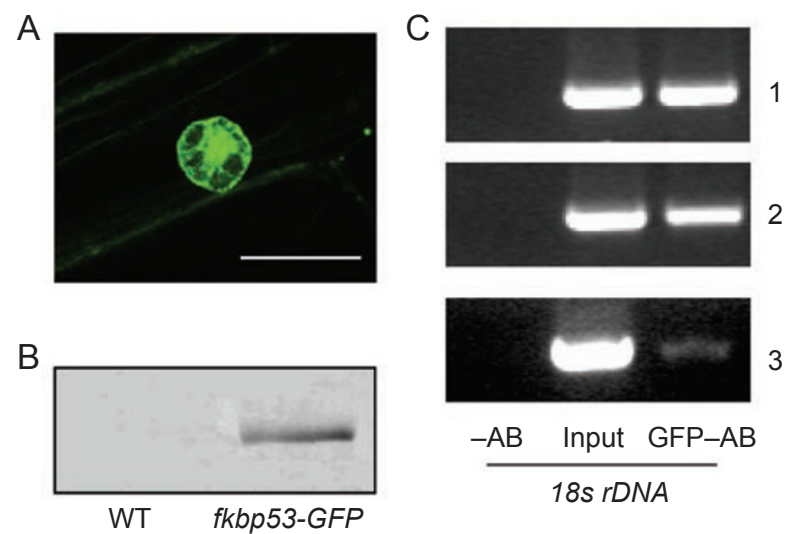

Figure 6 Association of AtFKBP53 with 18S rDNA chromatin. (A) AtFKBP53-GFP fluorescence. (B) Western blot analysis of total protein in wild-type and transgenic plants using anti-GFP antibody. (C) ChIP results from three independent transgenic lines of 35S-AtFKBP53-GFP. Samples were from leaves of 28-dayold transgenic plants. The input is chromatin before immunoprecipitation. 1-3, three lines of 35S-AtFKBP53-GFP plants; $-A B$, ChIP product with no antibody; GFP-AB, ChIP product with antibody to GFP. Scale bar, $10 \mu \mathrm{m}$ in (A). 
rDNA in vivo, whereas AtFKBP53 did not associate with other loci we examined, such as Knotted1-like (KNAT1), CYCLIN D $(C Y C D)$ and APETALA1 (APl) (Figure 6C; data not shown).

\section{Discussion}

We report that a nuclear FKBP-type immunophilin, AtFKBP53, is a functional histone chaperone that physically interacts with histone $\mathrm{H} 3$ and is possibly involved in $\mathrm{H} 3 / \mathrm{H} 4$ deposition to the nucleosome. Importantly, AtFKBP53 can interact with ribosomal DNA chromatin and regulate its gene expression, implicating AtFKBP53 in nucleosome assembly and gene regulation in plants.

The physiological functions of immunophilins, despite numerous biochemical analyses, are not well understood in plants or in other eukaryotic organisms. AtFKBP53 is a multidomain immunophilin that contains highly charged residues. We have shown here that AtFKBP53 functions as a histone chaperone through the acidic regions. Other histone chaperones reported to date all have acidic regions that are likely to be responsible for binding to histones that are highly basic proteins [30, 3337]. For example, both histone chaperones NAP-I and TAF-I interact with histones through an acidic region $[38,39]$. In addition, the acidic domains in these proteins are also sufficient for histone chaperone activity, indicating that these negatively charged domains also directly participate in the "assembly process" of nucleosomes. Further structure studies will reveal the acidic structure and elucidate the action mode of histone chaperones. It is noteworthy that Arabidopsis AtFKBP53 not only contains the acidic regions but also contains a basic domain. Therefore, we conclude that AtFKBP53, like SpFkbp39 in yeast, may represent a novel type of histone chaperone in plants. However, the function of the basic region in these proteins remains to be revealed.

AtFKBP53 can physically interact with histones, mainly with $\mathrm{H} 3$. The interaction with histone $\mathrm{H} 3$ is an indicator for the function in the formation of an $\mathrm{H} 3$ / H4 tetramer. Biochemical analysis has allowed for the separation of the nucleosome assembly reaction into two steps [40]. During the first step, the deposition of histones $\mathrm{H} 3$ and $\mathrm{H} 4$ to DNA occurs. The H3/H4 tetramer and DNA complex is a stable intermediate structure. In the second step, this preformed subnucleosome complex is converted to a mature nucleosome with the addition of two heterodimers of histones $\mathrm{H} 2 \mathrm{~A} / \mathrm{H} 2 \mathrm{~B}$. The binding of AtFKBP53 to histone H3 indicates that AtFKBP53 has a functional role in the first step, at least during formation of the nucleosome. Nevertheless, it is not ruled out that AtFKBP53 may function at the second step, because AtFKBP53 might interact with histones $\mathrm{H} 2 \mathrm{~A} / \mathrm{H} 2 \mathrm{~B}$, under less stringent conditions or certain circumstances.

Structural change of chromatin in eukaryotic cells influences biological processes such as gene expression. The structure of chromatin is maintained in part by histone chaperones. However, the functional specificity of various histone chaperones is not well understood. It is speculated that changes in chromatin structure often bring about global changes in expression of many genes. For example, AtCYP71 in plants clearly has such a global role and the cyp 71 mutant plants showed a number of developmental defects caused possibly by de-repression of many genes [31]. In this report, we show that AtFKBP53 associates with 18S rDNA chromatin and regulates the level of its expression. Significantly, the factors required for maintaining ribosomal gene expression remain largely unknown in plants. We therefore provide the first evidence showing that AtFKBP53 regulates $r R N A$ gene transcription at the chromatin level in plants.

It is puzzling why deregulation of rRNA gene expression did not cause visible changes in plant development. It is possible that upregulation of rRNA levels is not sufficient to change the functionality of ribosomes because other components may be limiting. Alternatively, some other chaperones may functionally overlap with AtFKBP53. One such candidate is AtFKBP43, another immunophilin protein that has a similar structure as AtFKBP53. AtFKBP43 may thus have similar functions as AtFKBP53. The $f k b p 43$ mutant, like $f k b p 53$ mutant, did not show obvious defects under normal conditions (Li et $a l$. , unpublished data). Further characterization of double mutant of $f k b p 53$ fkbp 43 may clarify the biological function of these immunophilins. In addition, further studies on functional interactions between AtFKBP53 and other histone chaperones may reveal more complicated relationship among these factors. Various histone chaperones may be involved in distinct and yet overlapping processes that maintain and change chromatin structure. Isolation of more histone chaperones will lead to a better understanding of the mechanism underlying nucleosome assembly and regulation of gene expression.

\section{Materials and Methods}

\section{Plant materials and growth conditions}

The seeds of $f k b p 53$ (GenBank accession number SALK 091242; background, Col) were provided by the Arabidopsis Biological Resource Center (ABRC). Plants were grown in a greenhouse at $22{ }^{\circ} \mathrm{C}$ with a photon flux density of $180 \mathrm{mmol} / \mathrm{m}^{2} / \mathrm{s}$ under a $16: 8 \mathrm{~h}$ light-dark cycle.

\section{Plasmid construction and plant transformation}

For protein expression, DNA fragments that encode for the AtFKBP53, as shown in Figure 2A, were PCR-amplified from the cDNA clone (U14536, from ABRC). Each forward primer car- 
ries a BamHI cutting site near its $5^{\prime}$ end, whereas each backward primer carries an XhoI cutting site near its $5^{\prime}$ end. For clones with N-terminal deletions (AtFKBP53-C1), a start codon was inserted following the coding sequence in the forward primer. These DNA fragments were digested with $B a m \mathrm{HI}$ and $\mathrm{XhoI}$, and cloned into the pGEX-4T-3 vector, which encodes a GST tag at the N terminus of each fusion protein. For protein localization transient expression cDNA of AtFKBP53 was cloned into pGDR vector by BglII and SalI enzyme sites. For transgenic plant, cDNA of AtFKBP53 without stop codon was cloned into pMD1 by $X b a 1$ and BamH1 sites. The constructs were transformed into Arabidopsis plants using the Agrobacterium tumefaciens-mediated floral dip procedure.

\section{Purification of proteins}

To purify AtFKBP53 and AtFKBP53 mutants, we introduced the expression vectors into Escherichia coli strain BL21. Cells were grown in LB medium supplemented with ampicillin $(100 \mu \mathrm{g} /$ $\mathrm{ml})$ at $37^{\circ} \mathrm{C}$ to OD600 0.5 and induced with $0.1 \mathrm{mM}$ isopropyl-Dthiogalactopyranoside at $37{ }^{\circ} \mathrm{C}$ for $4 \mathrm{~h}$. Cells from 1 liter cultures were then harvested and resuspended in $20 \mathrm{ml}$ PBS. The cell suspensions were sonicated and the lysates were subjected to centrifugation at $10000 \mathrm{rpm}$ for $20 \mathrm{~min}$. The supernatant was applied onto Glutathione Sepharose 4B beads prewashed with PBS. Purified proteins were resolved by SDS-PAGE and confirmed by western blot analysis.

\section{Histone chaperone assay}

The method was based on protocol described previously with slight modifications [7, 41]. We treated plasmid PUC19 (2 686 bp) with topoisomerase I (20 U/ $\mu$ g DNA; Invitrogen) for $30 \mathrm{~min}$. HeLa core histones ( 4 pmol) were preincubated with various proteins, including GST, SpFkbp39, AtFKBP53 and AtFKBP53 mutants for $15 \mathrm{~min}$, and then added the pretreated plasmid $(0.4 \mu \mathrm{g})$ into each of the chaperone-histone mixture and incubated for an additional $60 \mathrm{~min}$ in $50 \mu \mathrm{l}$ reaction buffer containing $0.15 \mathrm{M} \mathrm{NaCl}$, $0.5 \mathrm{mM}$ DTT and $20 \mathrm{mM}$ Tris- $\mathrm{HCl}(\mathrm{pH} 8.0)$, at $30^{\circ} \mathrm{C}$. The reactions were stopped by adding equal volume of stop buffer (glycerol 25\%, $60 \mathrm{mM}$ Tris- $\mathrm{HCl}$ (pH 8.0), $30 \mathrm{mM}$ EDTA, 0.5\% SDS) and were resolved in $1 \%$ agarose gel by electrophoresis followed by ethidium bromide staining.

\section{Protein-protein interaction}

In vitro pull-down assays were performed as described previously with modifications [31]. Purified recombinant GSTAtFKBP53, GST-AtFKBP53-N1, GST-AtFKBP53-N2, GSTAtFKBP53-C1 or GST $(1.5 \mu \mathrm{g})$ was immobilized to glutathione Sepharose beads at $4{ }^{\circ} \mathrm{C}$ for $2 \mathrm{~h}$ in the A300 binding buffer. After the beads were washed with A300 buffer three times, $1.5 \mu \mathrm{g}$ core histones (13-107; Upstate) was added and incubated for $4 \mathrm{~h}$ in A300 buffer. After the beads were extensively washed with A300 buffer, the histone $\mathrm{H} 3$ bound to the beads was determined by SDSPAGE and immunoblotting. A300 buffer contained $25 \mathrm{mM}$ Tris$\mathrm{HCl}$ (pH 8.0), 10\% glycerol, 0.01\% Nonidet P-40, 1 mM EDTA and $300 \mathrm{mM} \mathrm{NaCl}$. The histone $\mathrm{H} 3$ antibody (ab1791; Abcam) was used at dilution of 1:5 000 for protein gel blot analysis.

\section{RNA expression analysis}

Total RNA was extracted by Trizol from plant materials, and cDNA was synthesized by SuperScript II RNA polymerase. RT-
PCR was conducted using Taq polymerase to examine the expression levels of genes. Real-time PCR was performed according to previous methods [31].

\section{Detection of $\beta$-glucuronidase (GUS) activity}

Plants were grown under long day condition in the greenhouse. Plant tissue was placed in X-Gluc solution [1 mg/ml 5-bromo-4chloro-3-indolyl $\beta$-D-glucuronic acid (X-Gluc), $25 \mathrm{mM}$ phosphate buffer ( $\mathrm{pH} 7$ ), $1.25 \mathrm{mM}$ potassium ferricyanide, $1.25 \mathrm{mM}$ potassium ferrocyanide, $0.25 \mathrm{mM}$ EDTA and $0.25 \%$ Triton X-100] in a vacuum for $10 \mathrm{~min}$ at room temperature, and then incubated overnight at $37^{\circ} \mathrm{C}$, as previously described [42].

\section{Chromatin immunoprecipitation}

Chromatin immunoprecipitation (ChIP) experiments were performed as described previously with some modification [43]. Approximately $1.5 \mathrm{~g}$ rosette leaves of 28-day-old Arabidopsis plants grown on soil were fixed with $0.25 \%$ formaldehyde (EMD) under vacuum for $30 \mathrm{~min}$ until the leaf turns to be transparent. The reaction was stopped by the addition of glycine. The leaf material was ground in liquid nitrogen, and chromatin was extracted. The chromatin was sonicated into fragments ranging from $400 \mathrm{bp}$ to $1 \mathrm{kbp}$. The chromatin solution was precleared with Protein A agarose beads/salmon sperm DNA slurry (16-157; Upstate). The sonicated chromatin was immunoprecipitated with $8 \mu$ l of anti-GFP (Santa Cruz). Cross-linking was reversed in the immunoprecipitated complexes by the addition of $\mathrm{NaCl}$ to a final concentration of $200 \mathrm{mM}$ and incubation at $65^{\circ} \mathrm{C}$ for $6 \mathrm{~h}$ to overnight. The DNA was extracted from the immunoprecipitates by treatment with 20 $\mu \mathrm{g}$ proteinase $\mathrm{K}$, and purified by phenol-chloroform extraction and precipitation by ethanol and $15 \mathrm{mg} / \mathrm{ml}$ glycogen. Co-precipitated DNA was dissolved in $20 \mu \mathrm{l} \mathrm{TE}$, and typically $1 \mu \mathrm{l}$ was used for PCR analyses. DNA was amplified using primers specific to region of $18 \mathrm{~S}$ rDNA. Thirty cycles were used to amplify PCR fragment using Ex-Tag polymerase (Takara, Kyoto).

\section{Transient expression assays in onion epidermal cells}

For particle bombardment, all plasmids were amplified in $E$. coli strain and purified using plasmid midi or mini kits according to the manufacturer's instructions (Qiagen, Valencia, CA). Particle bombardment assays were performed on the epidermal peels from onion bulb scales. Onion epidermis was bombarded with gold particles coated with plasmids using a PDS-1000/He Biolistic particle delivery system Bio-Rad (Hercules, CA). Bombarded tissues were incubated in MS medium for 24-48 h in dark. Microscopy GFP and red fluorescence protein (RFP) were visualized using a Leica MZ FLIII fluorescence stereomicroscope equipped with GFP and RFP filters (Leica, Wetzlar, Germany) [44, 45].

\section{Accession numbers}

Sequence data for AtFKBP53 and $18 S$ rDNA can be found in GenBank under the nucleotide accession numbers At4G25340 and X16077, respectively.

\section{Acknowledgment}

We thank Veder Garcia (University of California, Berkeley, USA) for critically reading the paper, Zengyong He for providing the AtFKBP53::GUS transgenic line and Masami Horikoshi (The 
University of Tokyo, Japan) for the pET-6His-SpFkbp39P plasmid. This work was supported by grants from the National Science Foundation and US Department of Energy (to SL).

\section{References}

1 Bentley GA, Finch JT, Lewit-Bentley A, Roth M. The crystal structure of the nucleosome core particle by contrast variation. Basic Life Sci 1984; 27:105-117.

2 Richmond TJ, Finch JT, Rushton B, Rhodes D, Klug A. Structure of the nucleosome core particle at 7 A resolution. Nature 1984; 311:532-537.

3 Luger K, Mader AW, Richmond RK, Sargent DF, Richmond TJ. Crystal structure of the nucleosome core particle at $2.8 \mathrm{~A}$ resolution. Nature 1997; 389:251-260.

4 Dutta S, Akey IV, Dingwall C, et al. The crystal structure of nucleoplasmin-core: implications for histone binding and nucleosome assembly. Mol Cell 2001; 8:841-853.

5 Ito T, Bulger M, Kobayashi R, Kadonaga JT. Drosophila NAP-1 is a core histone chaperone that functions in ATPfacilitated assembly of regularly spaced nucleosomal arrays. Mol Cell Biol 1996; 16:3112-3124.

6 Rodriguez P, Munroe D, Prawitt D, et al. Functional characterization of human nucleosome assembly protein-2 (NAP1L4) suggests a role as a histone chaperone. Genomics 1997; 44:253-265.

7 Kuzuhara T, Horikoshi M. A nuclear FK506-binding protein is a histone chaperone regulating rDNA silencing. Nat Struct Mol Biol 2004; 11:275-283.

8 Loppin B, Bonnefoy E, Anselme C, et al. The histone H3.3 chaperone HIRA is essential for chromatin assembly in the male pronucleus. Nature 2005; 437:1386-1390.

9 Adams CR, Kamakaka RT. Chromatin assembly: biochemical identities and genetic redundancy. Curr Opin Genet Dev 1999; 9:185-190.

10 Angelov D, Bondarenko VA, Almagro S, et al. Nucleolin is a histone chaperone with FACT-like activity and assists remodeling of nucleosomes. Embo J 2006; 25:1669-1679.

11 Zhu Y, Dong A, Meyer D, et al. Arabidopsis NRP1 and NRP2 encode histone chaperones and are required for maintaining postembryonic root growth. Plant Cell 2006; 18:2879-2892.

12 Taneva SG, Banuelos S, Falces J, et al. A mechanism for histone chaperoning activity of nucleoplasmin: thermodynamic and structural models. J Mol Biol 2009; 393:448-463.

13 Daganzo SM, Erzberger JP, Lam WM, et al. Structure and function of the conserved core of histone deposition protein Asf1. Curr Biol 2003; 13:2148-2158.

14 Linger J, Tyler JK. The yeast histone chaperone chromatin assembly factor 1 protects against double-strand DNA-damaging agents. Genetics 2005; 171:1513-1522.

15 Kaya H, Shibahara KI, Taoka KI, et al. FASCIATA genes for chromatin assembly factor-1 in Arabidopsis maintain the cellular organization of apical meristems. Cell 2001; 104:131142.

16 March-Diaz R, Reyes JC. The beauty of being a variant: H2A. $\mathrm{Z}$ and the SWR1 complex in plants. Mol Plant 2009; 2:565577.

17 Choi K, Park C, Lee J, et al. Arabidopsis homologs of components of the SWR1 complex regulate flowering and plant development. Development 2007; 134:1931-1941.

18 Natsume R, Eitoku M, Akai Y, et al. Structure and function of the histone chaperone CIA/ASF1 complexed with histones H3 and H4. Nature 2007; 446:338-341.

19 Park YJ, Luger K. The structure of nucleosome assembly protein 1. Proc Natl Acad Sci USA 2006; 103:1248-1253.

20 Liu ZQ, Gao J, Dong AW, Shen WH. A truncated Arabidopsis NUCLEOSOME ASSEMBLY PROTEIN 1, AtNAP1;3T, alters plant growth responses to abscisic acid and salt in the Atnap1;3-2 mutant. Mol Plant 2009; 2:688-699.

21 Alexandre C, Moller-Steinbach Y, Schonrock N, Gruissem W, Hennig L. Arabidopsis MSI1 is required for negative regulation of the response to drought stress. Mol Plant 2009; 2:675687.

22 Shen $\mathrm{WH}, \mathrm{Xu}$ L. Chromatin remodeling in stem cell maintenance in Arabidopsis thaliana. Mol Plant 2009; 2:600-609.

$23 \mathrm{He}$ Y. Control of the transition to flowering by chromatin modifications. Mol Plant 2009; 2:554-564.

24 Georgel PT. Chromatin structure of eukaryotic promoters: a changing perspective. Biochem Cell Biol 2002; 80:295-300.

25 Eitoku M, Sato L, Senda T, Horikoshi M. Histone chaperones: 30 years from isolation to elucidation of the mechanisms of nucleosome assembly and disassembly. Cell Mol Life Sci 2008; 65:414-444.

26 Adkins MW, Howar SR, Tyler JK. Chromatin disassembly mediated by the histone chaperone Asfl is essential for transcriptional activation of the yeast $\mathrm{PHO5}$ and $\mathrm{PHO} 8$ genes. Mol Cell 2004; 14:657-666.

27 Korber P, Barbaric S, Luckenbach T, et al. The histone chaperone Asfl increases the rate of histone eviction at the yeast PHO5 and PHO8 promoters. J Biol Chem 2006; 281:55395545.

28 Zabaronick SR, Tyler JK. The histone chaperone anti-silencing function 1 is a global regulator of transcription independent of passage through S phase. Mol Cell Biol 2005; 25:652660.

29 Shan X, Xue Z, Melese T. Yeast NPI46 encodes a novel prolyl cis-trans isomerase that is located in the nucleolus. $J$ Cell Biol 1994; 126:853-862.

30 Xiao H, Jackson V, Lei M. The FK506-binding protein, Fpr4, is an acidic histone chaperone. FEBS Lett 2006; 580:43574364.

31 Li H, He Z, Lu G, et al. A WD40 domain cyclophilin interacts with histone $\mathrm{H} 3$ and functions in gene repression and organogenesis in Arabidopsis. Plant Cell 2007; 19:2403-2416.

32 He Z, Li L, Luan S. Immunophilins and parvulins. Superfamily of peptidyl prolyl isomerases in Arabidopsis. Plant Physiol 2004; 134:1248-1267.

33 Okuwaki M, Matsumoto K, Tsujimoto M, Nagata K. Function of nucleophosmin/B23, a nucleolar acidic protein, as a histone chaperone. FEBS Lett 2001; 506:272-276.

34 Erard MS, Belenguer P, Caizergues-Ferrer M, Pantaloni A, Amalric F. A major nucleolar protein, nucleolin, induces chromatin decondensation by binding to histone H1. Eur J Biochem 1988; 175:525-530.

35 Shintomi K, Iwabuchi M, Saeki H, et al. Nucleosome assembly protein-1 is a linker histone chaperone in Xenopus eggs. Proc Natl Acad Sci USA 2005; 102:8210-8215.

36 Lee IS, Oh SM, Kim SM, Lee DS, Seo SB. Highly acidic 
C-terminal domain of pp32 is required for the interaction with histone chaperone, TAF-Ibeta. Biol Pharm Bull 2006; 29:2395-2398.

37 Silverman BD. Hydrophobic and acidic moments of a nucleoplasmin NP-core chaperone. J Biomol Struct Dyn 2006; 24:49-56.

38 Oeda K, Salinas J, Chua NH. A tobacco bZip transcription activator (TAF-1) binds to a G-box-like motif conserved in plant genes. EMBO J 1991; 10:1793-1802.

39 Ishimi Y, Kikuchi A. Identification and molecular cloning of yeast homolog of nucleosome assembly protein I which facilitates nucleosome assembly in vitro. J Biol Chem 1991; 266:7025-7029.

40 Smith S, Stillman B. Stepwise assembly of chromatin during DNA replication in vitro. EMBO J 1991; 10:971-980.

41 Terakura S, Ueno Y, Tagami H, et al. An oncoprotein from the plant pathogen agrobacterium has histone chaperone-like activity. Plant Cell 2007; 19:2855-2865.

42 Ori N, Eshed Y, Chuck G, Bowman JL, Hake S. Mechanisms that control knox gene expression in the Arabidopsis shoot. Development 2000; 127:5523-5532.

43 Gendrel AV, Lippman Z, Yordan C, Colot V, Martienssen RA. Dependence of heterochromatic histone H3 methylation patterns on the Arabidopsis gene DDM1. Science 2002; 297:1871-1873.

44 Kuijt SJ, Lamers GE, Rueb S, et al. Different subcellular localization and trafficking properties of KNOX class 1 homeodomain proteins from rice. Plant Mol Biol 2004; 55:781796.

$45 \mathrm{Xu} \mathrm{J}$, Scheres B. Dissection of Arabidopsis ADP-RIBOSYLATION FACTOR 1 function in epidermal cell polarity. Plant Cell 2005; 17:525-536. 